\title{
A DETERMINATION OF THE SPIN-ORBIT ALIGNMENT OF THE ANOMALOUSLY DENSE PLANET ORBITING HD $149026^{1}$
}

\author{
Aaron S. Wolf, ${ }^{2,3}$ Gregory Laughlin, ${ }^{2}$ Gregory W. Henry, ${ }^{4}$ \\ Debra A. Fischer, ${ }^{5}$ Geoff Marcy, ${ }^{6}$ Paul Butler, ${ }^{7}$ and Steve Vogt ${ }^{2}$ \\ Received 2005 November 1; accepted 2006 February 11
}

\begin{abstract}
We report 35 radial velocity measurements of HD 149026 taken with the Keck Telescope. Of these measurements, 15 were made during the transit of the companion planet HD 149026b, which occurred on 2005 June 25. These velocities provide a high-cadence observation of the Rossiter-McLaughlin effect, the shifting of photospheric line profiles that occurs when a planet occults a portion of the rotating stellar surface. We combine these radial velocities with previously published radial velocity and photometric data sets and derive a composite best-fit model for the star-planet system. This model confirms and improves previously published orbital parameters, including the remarkably small planetary radius, the planetary mass, and the orbital inclination, found to be $R_{p} / R_{\text {Jup }}=0.718 \pm 0.065, M_{p} / M_{\text {Jup }}=0.352 \pm 0.025$, and $I=86.1^{\circ} \pm 1.4^{\circ}$, respectively. Together the planetary mass and radius determinations imply a mean planetary density of $1.18_{-0.30}^{+0.38} \mathrm{~g} \mathrm{~cm}^{-3}$. The new data also allow for the determination of the angle between the apparent stellar equator and the orbital plane, which we constrain to be $\lambda=-12^{\circ} \pm 15^{\circ}$.
\end{abstract}

Subject headings: planetary systems — planets and satellites: general — stars: individual (HD 149026)

\section{INTRODUCTION}

The transit of an extrasolar giant planet across the face of a bright parent star is a rare and valuable phenomenon. Accordingly, it is quite fortunate that with each transit found, a wealth of information is uncovered. Indeed, dozens of research papers begin by recounting a now familiar litany: careful observation of transits can yield direct measurements of the planetary size, density, composition, mass, temperature, and albedo. For an overview of follow-up observational techniques for studying transiting extrasolar planets, see Charbonneau 2004). Furthermore, models that combine photometry with radial velocity measurements can greatly refine the orbital parameters (see, e.g., Pont et al. 2004; Bouchy et al. 2004; Moutou et al. 2004; Holman et al. 2007; Wittenmyer et al. 2005; Winn et al. 2005; Laughlin et al. 2005) and can lead to the determination of such quantities as the alignment of the orbital angular momentum vector with the spin axis of the star (e.g., Winn et al. 2005).

In addition to informing us with physical facts, the transiting planets also provide insight into the formation and evolutionary processes that fashioned the galactic planetary census. For example, the small $\left(0.725 R_{\text {Jup }}\right)$ observed radius of the $0.36 M_{\text {Jup }}$ transiting planet HD 149026b (Sato et al. 2005) provides solid evidence that the core accretion process plays a role in giant planet formation (e.g., Pollack et al. 1996; Hubickyj et al. 2004). That is, interior models of HD 149026b (Sato et al. 2005; Fortney et al. 2005 ) indicate that more than $50 \%$ of the planetary mass is in the

\footnotetext{
${ }^{1}$ Based on observations obtained at the W. M. Keck Observatory, which is operated jointly by the University of California and the California Institute of Technology.

${ }^{2}$ UCO/Lick Observatory, University of California, Santa Cruz, CA 95064.

${ }^{3}$ Geological and Planetary Sciences, California Institute of Technology, Pasadena, CA 91125

${ }^{4}$ Center of Excellence in Information Systems, Tennessee State University, Nashville, TN 37209.

${ }^{5}$ Department of Physics and Astronomy, San Francisco State University, San Francisco, CA 94132.

${ }^{6}$ Department of Astronomy, University of California, Berkeley, CA 94720.

${ }^{7}$ Department of Terrestrial Magnetism, Carnegie Institute of Washington, Washington, DC 20015-1305.
}

form of elements heavier than hydrogen and helium. Unlike with core accretion theory, a metal-dominated composition is inconsistent with the gravitational instability mechanism for giant planet formation (e.g., Boss 1997, 2000, 2003).

In this paper we report and discuss follow-up radial velocity observations of HD 149026 that were scheduled to sample a full transit. As the planet passes in front of the star, it produces a characteristic radial velocity signature that arises from occultation of part of the rotating stellar surface. This phenomenon was first measured for the eclipsing binary stars $\beta$ Lyrae (Rossiter 1924) and Algol (McLaughlin 1924) and was first measured for an eclipsing extrasolar planet during the transit of HD 209458 by Queloz et al. (2000) and Bundy \& Marcy (2000). Recently, Winn et al. (2005) made a careful analysis of the full 85 point radial velocity data set for HD 209458 reported by Laughlin et al. (2005), which includes 19 spectra taken during transits. Winn et al. (2005) showed that HD 209458 exhibits an effective radial velocity halfamplitude of $\Delta v=55 \mathrm{~m} \mathrm{~s}^{-1}$ during the eclipse, indicating a lineof-sight rotation speed of the star of $v \sin I_{\star}=4.70 \pm 0.16 \mathrm{~km} \mathrm{~s}^{-1}$. A small asymmetry appears in the in-transit radial velocity curve and is modeled as the result of an inclination of the planetary orbit relative to the apparent stellar equator of $\lambda=-4.4^{\circ} \pm 1.4^{\circ}$. For comparison, the degree of misalignment in our own system, between the solar rotation axis and the net orbital angular momentum vector of the planets, is about $7^{\circ}$. In this paper our primary aim is to determine the degree to which the stellar spin axis and the planetary orbital angular momentum vector are aligned for HD 149026 and its planetary companion. A significant misalignment can be indicative of a complex formation history for the system, which might provide additional constraints in trying to understand this strange object. Such a history is of particular relevance for HD 149026b, because a collision between large giant-planet embryos is a possible mechanism for forming a heavyelement-dominated planet. Alternatively, a near alignment is suggestive of a more quiescent evolution, dominated by interactions with a protoplanetary disk.

The plan of this paper is as follows. In $\S 2$ we describe the radial velocity and photometric data sets that we use in our analysis. In $\S 3$ we discuss the details of our model of the system. In $\S 4$ we 
discuss the details of our fitting and error estimation process as well as present our results, and in $\oint 5$ we discuss their ramifications.

\section{THE DATA}

Our analysis uses two general types of data: (1) radial velocity measurements obtained for the star both during transit and out of transit and (2) differential photometry obtained during six different transits. Our radial velocity data set contains all the measurements reported by Sato et al. (2005), as well as a number of new radial velocities obtained both in and out of transit. Our photometric data includes the measurements published by Sato et al. (2005), as well as those reported by Charbonneau et al. (2006).

\subsection{Radial Velocities}

As described in Sato et al. (2005), four N2K program (Fischer et al. 2005) Doppler observations taken at Subaru flagged the metal $\mathrm{rich}[\mathrm{Fe} / \mathrm{H}]=0.36, V=8.15, \mathrm{G} 0 \mathrm{IV}$ star HD 149026 as a planet-bearing candidate. Velocities derived from seven follow-up observations at Keck were used to determine the orbital parameters and a predicted ephemeris time for transit observations. Three of the initial seven Keck observations were serendipitously obtained during transits, with another point just outside of transit, already revealing the Rossiter-McLaughlin effect. The Keck observations were made with the High Resolution Echelle Spectrometer (Vogt et al. 1994) with a spectral resolution of about 55,000 and typical signal-to-noise ratio of 250 . The Doppler analysis makes use of an iodine cell in the light path as described by Butler et al. (1996).

In the present analysis, we include a total of 35 Doppler measurements from Keck, plus the initial four radial velocity (RV) measurements from Subaru. All of the 35 Keck RV measurements, including the original seven reported by Sato et al. (2005), have been reanalyzed by obtaining a deeper template spectrum of HD 149026 and are listed relative to a new velocity zero point in Table 1.

\subsection{Transit Photometry}

Our transit photometry comes from two different sources. The data for the first three transits are from Sato et al. (2005), who described the observational configuration in detail. These measurements were taken at the Fairborn Observatory during 2005 May and June. The last of these three Fairborn data sets is a binned compilation of the observations of a single transit by three separate telescopes, again reported in the photometry data section of Sato et al. (2005). As a result of binning, the photometric points in this composite data set have relative uncertainties that are smaller by a factor of about $\sqrt{3}$ in comparison to the first two data sets. The uncertainties for all photometric data points within a given transit night are determined by the standard deviation of all outof-transit points from baseline, just as in Sato et al. (2005).

The additional three data sets are from Charbonneau et al. (2006). These observations were made using a series of different filters and together represent two partial to nearly full transits. Due to the strong effect of increasing air mass within these data sets, it was necessary for Charbonneau et al. (2006) to individually determine the proper weighting factor for the data points within each portion of the transit. This was achieved by a more sophisticated method than that described above, which accounts for photometric variability caused by the combination of Poisson photon statistics and nonnegligible air mass. We therefore adopt their weights as estimates of photometric error.

\section{MODEL DESCRIPTION}

Our analysis assumes that the system consists of a single planet on a Keplerian orbit, which is coupled to a stellar flux model
TABLE 1

Keck Radial Velocity Observations of HD 149026

\begin{tabular}{|c|c|c|}
\hline HJD & Radial Velocities & $\begin{array}{c}\text { Uncertainties } \\
\quad\left(\mathrm{m} \mathrm{s}^{-1}\right)\end{array}$ \\
\hline $2453427.15862 \ldots \ldots \ldots \ldots$ & 2.41 & 3.21 \\
\hline $2453429.11393 \ldots \ldots \ldots . .$. & 28.97 & 3.47 \\
\hline $2453430.07941 \ldots \ldots \ldots . .$. & 3.17 & 3.38 \\
\hline $2453430.09579 \ldots \ldots \ldots \ldots$ & -4.29 & 3.29 \\
\hline $2453479.03648 \ldots \ldots \ldots \ldots$ & -13.36 & 4.20 \\
\hline $2453480.11060 \ldots \ldots \ldots \ldots$ & -21.33 & 3.20 \\
\hline $2453483.93001 \ldots \ldots \ldots . .$. & 35.50 & 4.06 \\
\hline $2453546.82987 \ldots \ldots \ldots . .$. & 8.55 & 3.05 \\
\hline $2453547.78164 \ldots \ldots \ldots . .$. & 15.37 & 2.36 \\
\hline $2453547.82296 \ldots \ldots \ldots . .$. & 9.19 & 2.59 \\
\hline $2453547.85172 \ldots \ldots \ldots . .$. & 10.95 & 1.98 \\
\hline $2453547.88439 \ldots \ldots \ldots . .$. & 7.67 & 2.15 \\
\hline $2453547.89184 \ldots \ldots \ldots . .$. & 5.89 & 2.10 \\
\hline $2453547.91875 \ldots \ldots \ldots . .$. & -0.72 & 1.81 \\
\hline $2453547.93571 \ldots \ldots \ldots . .$. & 2.10 & 2.14 \\
\hline $2453547.95362 \ldots \ldots \ldots \ldots$ & 13.59 & 1.65 \\
\hline $2453547.96604 \ldots \ldots \ldots \ldots$ & 10.81 & 2.09 \\
\hline $2453547.97235 \ldots \ldots \ldots \ldots$ & 13.35 & 2.38 \\
\hline $2453547.98637 \ldots \ldots \ldots \ldots$ & 8.42 & 1.97 \\
\hline $2453547.99556 \ldots \ldots \ldots . .$. & 2.04 & 2.01 \\
\hline $2453548.00679 \ldots \ldots \ldots \ldots$ & -6.24 & 1.96 \\
\hline $2453548.01510 \ldots \ldots \ldots \ldots$ & -14.99 & 1.84 \\
\hline $2453548.02714 \ldots \ldots \ldots . .$. & -16.15 & 1.88 \\
\hline $2453548.03490 \ldots \ldots \ldots \ldots$ & -12.39 & 3.88 \\
\hline $2453548.04598 \ldots \ldots \ldots . .$. & -18.94 & 3.33 \\
\hline $2453548.05911 \ldots \ldots \ldots . .$. & -19.69 & 2.50 \\
\hline $2453548.06399 \ldots \ldots \ldots . .$. & -6.20 & 3.27 \\
\hline $2453548.07928 \ldots \ldots \ldots \ldots$ & -5.13 & 2.69 \\
\hline $2453548.93858 \ldots \ldots \ldots \ldots$ & -43.02 & 1.95 \\
\hline $2453549.83153 \ldots \ldots \ldots$ & 22.55 & 3.14 \\
\hline $2453550.86215 \ldots \ldots \ldots \ldots$ & 0.43 & 2.14 \\
\hline $2453551.87838 \ldots \ldots \ldots . .$. & -44.63 & 2.08 \\
\hline $2453570.85661 \ldots \ldots \ldots \ldots$ & 15.41 & 2.80 \\
\hline $2453575.93859 \ldots \ldots \ldots .$. & 33.86 & 4.02 \\
\hline $2453602.74500 \ldots \ldots \ldots \ldots$ & -23.22 & 3.22 \\
\hline
\end{tabular}

arising from a spherical, nonvariable star. Due to the presence of the Rossiter-McLaughlin effect within the RV observations, nearly all the parameters necessary to describe the complete model are shared to some degree between the RVs and the photometry; the exceptions are the data offset parameters, which are necessarily specific to each component of the model, and the stellar spin parameters specific to describing the Rossiter-McLaughlin effect.

\subsection{The Orbit}

We choose to restrict our model to zero-eccentricity orbits. This decision is made for two reasons: (1) the out-of-transit RV observations are well described by a simple sinusoid, eliminating the need to introduce orbital eccentricity as a fitting parameter, as is the case for HD 209458 (Laughlin et al. 2005; Winn et al. 2005); and (2) a zero eccentricity orbit is expected for a short-period single planet system due to a short tidal circularization timescale, which for this system, assuming a planet with $Q=200,000$, is only $\tau_{\text {circ }}=150 \mathrm{Myr}$ (Bodenheimer et al. 2003). The parameters that we vary include the period, $P$, the stellar mass, $M_{0}$, the planetary mass, $M_{p}$, and the orbital inclination, $I$. We also allow for a uniform total velocity offset $\left(\nu_{\text {tot }}\right)$ and a relative velocity offset between the Keck and Subaru telescopes $\left(\nu_{\text {rel }}\right)$. Together, these two offset parameters adjust for overall and relative shifts in the 
velocity zero points of the two data sets. We choose to parameterize the initial starting condition in terms of the mean anomaly $\left(M_{0}\right)$ at the reported epoch of central transit for the 2005 June 5 transit from Charbonneau et al. (2006), $t_{0}=$ HJD 2,453,527.87455. We have chosen this reference epoch because it represents one of the most well constrained portions of the photometric data series, situated roughly at the center of the observing baseline and receiving independent coverage from multiple separate observations. For this model, we employ the standard Cartesian coordinate system in which edge-on orbits are confined to the $x-y$ plane: the $y$-axis lies along the radial vector pointing from the earth to the system, the $x$-axis lies in the plane of the sky, and the $z$-axis is the mutually perpendicular vector completing the set. Using this coordinate system, we calculate the orbital component of the RV variations by sampling the negative $y$-component of the stellar velocity $(-\dot{Y})$.

\subsection{The Flux}

The second portion of the model involves determining the time-dependent decrease in stellar flux that occurs while the planet is in transit. As the planet occults different portions of the stellar disk, the amount of light that it blocks changes in response to the effect of limb darkening. We employ the standard linear limbdarkening relation to model the intensity,

$$
I(\mu)=1-u(1-\mu)
$$

where $\mu$ is the cosine of the angle between the line of sight and the local normal to the stellar surface, and $u$ is the appropriate limb-darkening coefficient. We employ the limb darkening coefficients reported in Charbonneau et al. (2006) for each of the photometric data sets and use an average of these values, which correspond to observations made in the visible range, to obtain an appropriate limb-darkening value for the Rossiter-McLaughlin observations:

$u_{g}=0.73, u_{r}=0.61, u_{V}=0.62, u_{b+y}=0.67, u_{\text {Ross }}=0.66$,

where $u_{g}, u_{r}$, and $u_{V}$ apply to the $g$-filter, $r$-filter, and $V$-filter photometry reported in Charbonneau et al. (2006); $u_{b+y}$ applies to the $(b+y)$-filter photometry reported in Sato et al. (2005); and $u_{\text {Ross }}$ applies to the transit modeling of the Rossiter-McLaughlin effect within the RVs.

In order to determine the total flux, we assume that the planet is an opaque circular disk, and we integrate equation (1) over the unobscured portion of the stellar disk. In order to accomplish this with minimal computational effort, we employ the approximate analytic integrals given by Ohta et al. (2005). With these formulas, the diminished stellar flux is rapidly calculated as a function of the planet's size and position. The parameters relevant to the transit curve are the period, $P$; stellar mass, $M_{\star} ;$ stellar radius, $R_{\star} ;$ planetary radius, $R_{p}$; and orbital inclination, $I$. In analogy to the RV offset parameters described above, $\nu_{\text {tot }}$ and $\nu_{\text {rel }}$, we also allow for an overall magnitude offset parameter $\left(\mu_{\text {tot }}\right)$, as well as relative magnitude offset parameters for each of the five remaining photometric data sets $\left(\mu_{1}, \ldots, \mu_{5}\right)$. It is most natural to define the initial starting condition in terms of the central transit time, $T_{c}$, which can be determined from the mean anomaly using

$$
T_{c}=t_{0}+P\left(M_{0}-90^{\circ}\right) / 360^{\circ},
$$

where $t_{0}=2,453,527.87455$ is the epoch for which $M_{0}$ is defined.

\subsection{The Rossiter-McLaughlin Effect}

A circular planetary orbit is fully adequate to describe all of the out-of-transit RV variations. During transit, there is an additional contribution, however, resulting from the transiting planetary disk differentially obscuring portions of the rotating star. This socalled Rossiter-McLaughlin effect causes a characteristic "S-wave" shaped shift in the RV curve as the planet crawls across the stellar disk. If the planet's orbit is prograde relative to the stellar spin, the direction of the Rossiter shift is always positive and then negative: on ingress, the planet covers the oncoming stellar limb, blocking out blueshifted light, while on egress it covers the retreating limb, blocking out redshifted light. The Doppler velocity modeling code (see Butler et al. 1996), which transforms spectral line shifts into $\mathrm{RVs}$, interprets the resulting line profile asymmetries as RV shifts, resulting in the curve depicted in Figure 1. In order to model this curve, we again use the analytic approximate integrals reported in Ohta et al. (2005). These formulas provide the Rossiter velocity shift, $\Delta v_{\text {Ross }}$, as a function of the stellar rotation speed, and the planet's size and position.

With the exception of the photometric and velocity offset parameters, the variables needed to fully describe the RossiterMcLaughlin effect include all of the parameters already employed for both the orbital and transit models: namely, $P, M_{\star}, R_{\star}, M_{p}, R_{p}$, and $I$. Two additional parameters are also required. These are (1) the projection of the stellar rotation velocity onto the plane of the sky, $v \sin I_{\star}$, which scales the amplitude of the variations; and (2) the projection, $\lambda$, of the spin-orbit misalignment angle onto the plane of the sky, which determines the asymmetry of the Rossiter curve. Note that $v \sin I_{\star}$ was determined by Sato et al. (2005) from spectral line broadening to be $v \sin I_{\star}=6.0 \pm$ $0.5 \mathrm{~km} \mathrm{~s}^{-1}$. Due to the Rossiter curve's amplitude dependence on the stellar rotation speed, in-transit RV observations provide the opportunity to obtain an independent estimate of $v \sin I_{\star}$, and thus we choose to include $v \sin I_{\star}$ as a free parameter of the model. If the axes of the planetary orbit and stellar spin are perfectly aligned with $\lambda=0$, then the transiting planet crosses the RV zero point exactly halfway through the transit. If the misalignment angle is not zero, however, noncentral transits will cross this zero-velocity point either earlier or later. In addition, the amplitude on either side of the zero-velocity point is affected for noncentral transits, as the planet can potentially cover faster or slower portions of the stellar limb, depending on the degree of misalignment. As described previously, we parameterize the initial starting condition for the Rossiter curve in terms of the mean anomaly $M_{0}$ at the epoch HJD 2,453,527.87455, using equation (2) to convert between $M_{0}$ and the more natural parameterization for transits in terms of the central transit time.

\section{FITTING PROCEDURE AND RESULTS}

Our model has 17 free parameters. Eight of the parameters describe physically significant quantities, one of them parameterizes the initial starting condition, and the remaining eight account for baseline offsets among the individual data sets. While there are eight physically significant variables, not all of them are well constrained by the data we possess. There is a well-known degeneracy between $M_{\star}, M_{p}$, and $R_{\star}$ when their values must be determined from transit data alone, and furthermore, information about $R_{\star}$ is contained almost exclusively in the subtle shape of the transit light curve during ingress and egress. We therefore fix the values of the stellar mass and radius to be $M_{\star}=1.30 M_{\odot}$ and $R_{\star}=$ $1.45 R_{\odot}$, in accordance with the values obtained from stellar parallax and spectral modeling (Sato et al. 2005), providing additional and necessary constraints on these parameters. Using a 

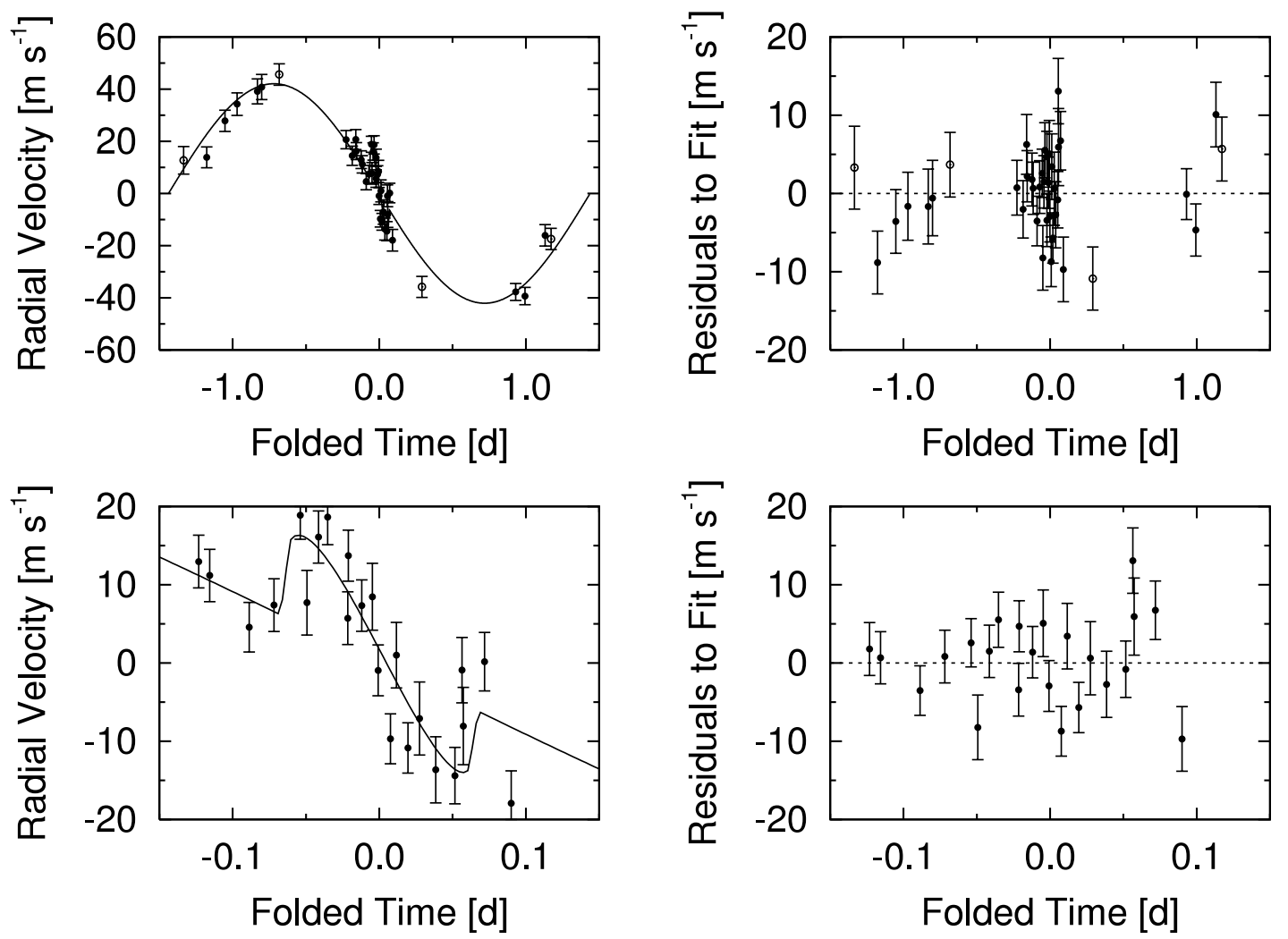

FIG. 1.-Comparison of RV data and best-fit model. All data points have been folded about the transit center. Open circles represent Subaru observations, and filled circles represent Keck observations. Top: All folded RV data plus model. Bottom: Close-up of RV points near transit phase.

Levenburg-Marquardt minimization scheme similar to that described in Press et al. (1992), we fit for the remaining 15 free parameters by minimizing:

$$
\chi^{2}=\sum_{i=1}^{N_{v}=39}\left(\frac{v_{o}-v_{c}}{\sigma_{v}}\right)^{2}+\sum_{i=1}^{N_{f}=2304}\left(\frac{f_{o}-f_{c}}{\sigma_{f}}\right)^{2},
$$

where $v_{o}$ and $v_{c}$ are the observed and calculated RVs, of which there are $N_{v}=39$, and $f_{o}$ and $f_{c}$ are the observed and calculated stellar fluxes, of which there are $N_{f}=2304$. The photometric uncertainties $\sigma_{f}$ used in this equation are obtained as described above. The RV uncertainties, $\sigma_{v}$, are those reported in Table 1 and in Sato et al. (2005) for Keck and Subaru velocities, respectively, with an additional $2.6 \mathrm{~m} \mathrm{~s}^{-1}$ of expected stellar jitter added in quadrature (Saar et al. 1998; Wright et al. 2004; Sato et al. 2005).

Using this method, we obtain a best fit with a reduced $\chi^{2}$ value, $\chi_{r}^{2}=1.00007=\chi^{2} / N_{\text {dof }}$, where $N_{\text {dof }}=N_{f}+N_{v}-15=2328$ is the number of degrees of freedom. An acceptable fit is expected to have $\chi_{r}^{2} \approx 1.0$, and thus we find our best fit to be a fully adequate model of the data. The parameter values for this model are shown in Table 2, and the corresponding RV and photometric models are shown in Figures 1 and 2.

In order to estimate the uncertainties in each of these parameters, we performed a bootstrap Monte Carlo analysis as described by Press et al. (1992). We created synthetic RV and photometric data sets by randomly redrawing values from the actual data sets (with replacement). We required that the total number of photometric and RV data points remain constant. ${ }^{8}$ Each of these syn-

\footnotetext{
${ }^{8}$ We also insist that at least one data point be represented from each of the eight observational data sets; this is required by the Levenburg-Marquardt method such that fits do not become degenerate in the offset parameters.
}

thetic data sets were then fitted using the method described above, where the initial guesses for the Levenburg-Marquardt method were obtained by taking small Gaussian-random deviations from the best-fit values for each of the parameters. In addition, we included the uncertainty in the stellar mass and radius by fixing the value of each to a Gaussian random number drawn from distributions based on their parallax and spectrally estimated values of

TABLE 2

Planetary and Stellar Properties for HD 149026b

\begin{tabular}{|c|c|}
\hline Parameter & Value \\
\hline 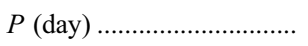 & $2.87618_{-0.00033}^{+0.00018}$ \\
\hline 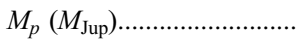 & $0.352 \pm 0.025$ \\
\hline 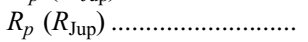 & $0.718 \pm 0.065$ \\
\hline$I(\operatorname{deg})$ & $86.1 \pm 1.4$ \\
\hline 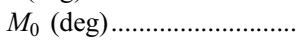 & $90.04_{-0.03}^{+0.55}$ \\
\hline$M_{\star}\left(M_{\odot}\right) \ldots \ldots \ldots \ldots \ldots \ldots \ldots \ldots$ & $1.30 \pm 0.10$ \\
\hline 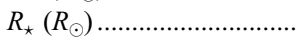 & $1.45 \pm 0.10$ \\
\hline 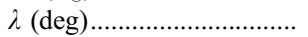 & $-12 \pm 15$ \\
\hline$v \sin I_{\star}\left(\mathrm{km} \mathrm{s}^{-1}\right) \ldots \ldots \ldots \ldots \ldots$ & $6.2_{-0.6}^{+2.1}$ \\
\hline$A_{\text {ratio }}=\left(R_{p} / R_{\star}\right)^{2} \ldots \ldots \ldots \ldots \ldots$ & $0.00257 \pm 0.00015$ \\
\hline 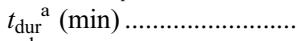 & $195 \pm 5$ \\
\hline$T_{c}^{\mathrm{b}}(\mathrm{HJD}) \ldots \ldots \ldots \ldots \ldots \ldots \ldots \ldots \ldots \ldots \ldots \ldots \ldots$ & $2453527.872 \pm 0.002$ \\
\hline
\end{tabular}

a The transit duration, $t_{\text {dur }}$, refers to the time between the points of first and last contact.

$\mathrm{b}$ The reported transit center, $T_{c}$, is the time of central transit that is most well constrained by the data. This corresponds to the center of the 2005 June 5 transit, observed independently by Sato et al. (2005) and Charbonneau et al. (2006). Here we report a symmetric error estimate, although there is a small branch of non-Gaussian early transit solutions, as described in the text. 

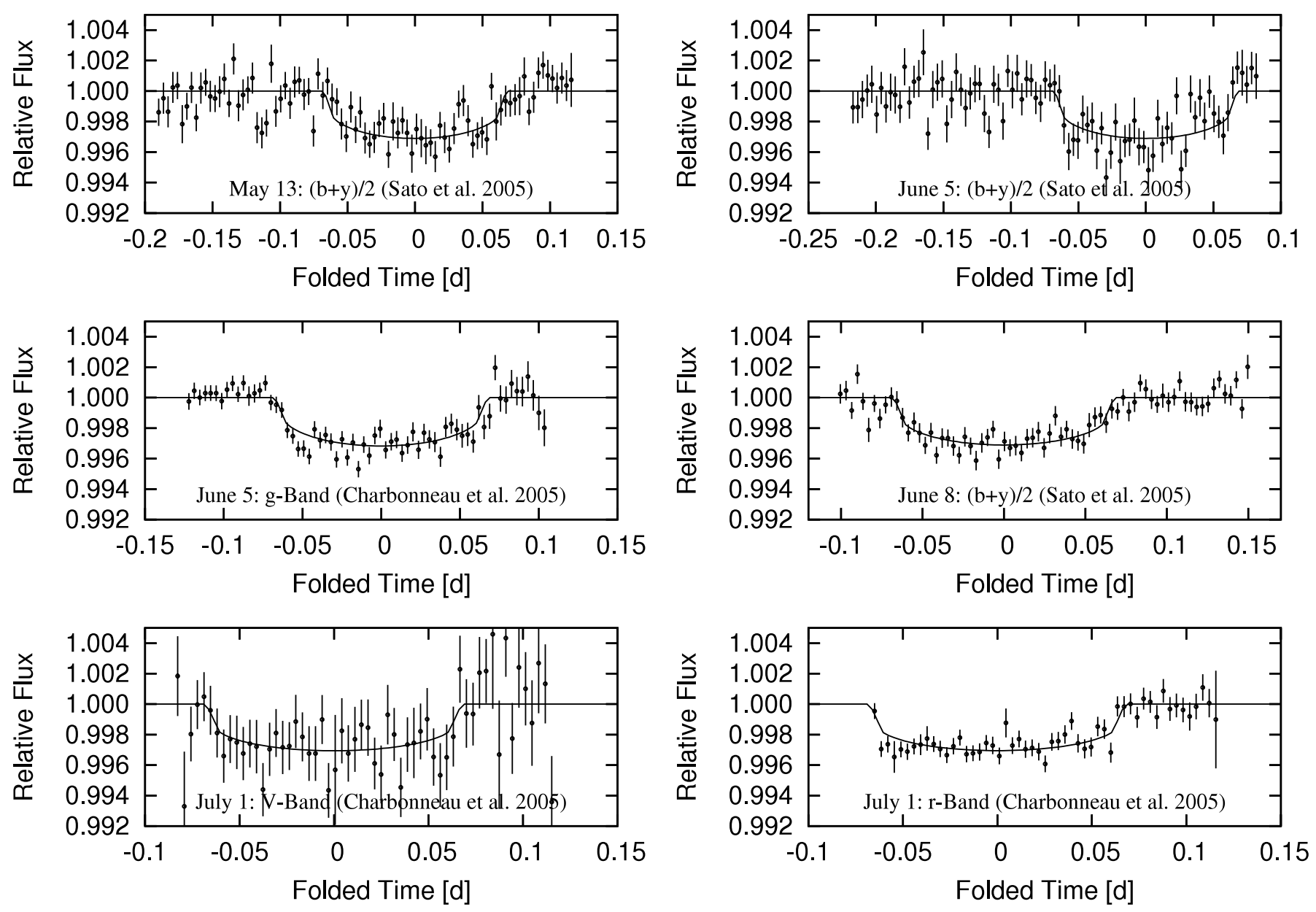

FIG. 2.-Comparison of six observed transits and best-fit photometric models. Slight variations in transit shape result from the different limb-darkening values appropriate to each observation. These points are displayed using 5 minute time bins for presentation purposes, although all analyses were performed on the unbinned data.

$R_{\star}=1.45 \pm 0.10 R_{\odot}$ and $M_{\star}=1.30 \pm 0.10 M_{\odot}$. As noted by Charbonneau et al. (2006), this does not account for the known correlation between stellar mass and radius. We choose, however, not to assume a mass-radius relation, as it depends on the evolutionary state of the star, which is highly uncertain for subgiants such as HD 149026. If we were able to use the proper

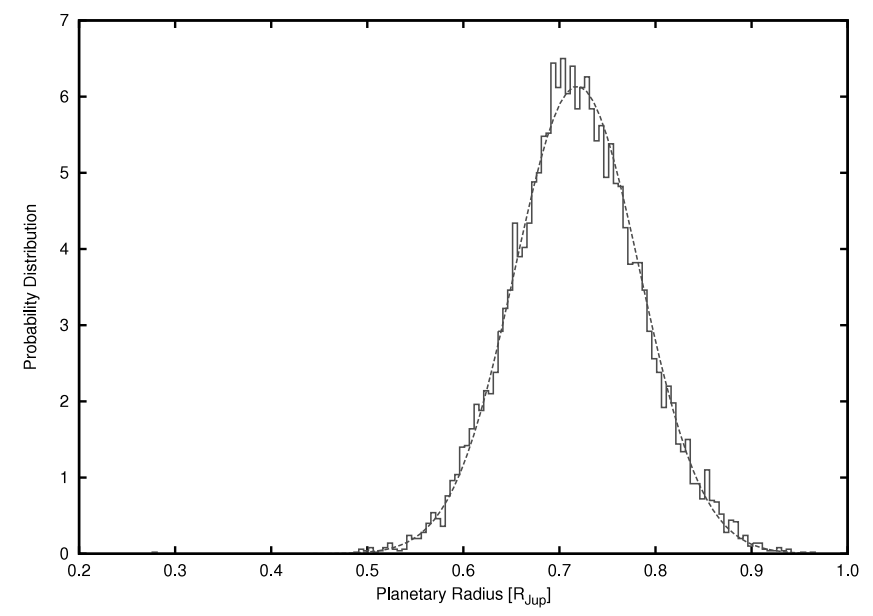

FIG. 3.-Probability distribution for planetary radius, generated from $10^{4}$ bootstrap Monte Carlo results. Also plotted is the best-fit Gaussian, indicating a planetary radius of $R_{p}=(0.718 \pm 0.065) R_{\text {Jup }}$. relation, it would slightly reduce our reported uncertainties. The bootstrap procedure was repeated $10^{4}$ times, in order to build smooth statistical distributions. In Table 2 we report the $1 \sigma$ error bars for each parameter, as determined from the best-fit Gaussian to the distribution of results from the bootstrap analysis. Also reported is the area ratio between star and planet, $A_{\text {ratio }}$, the

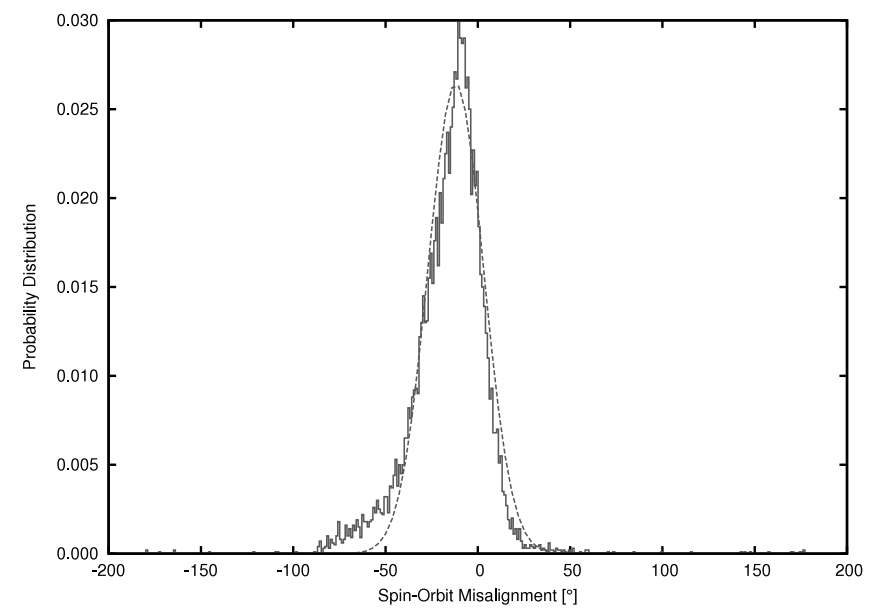

Fig. 4.-Probability distribution for spin-orbit misalignment, as determined from $10^{4}$ bootstrap Monte Carlo results. Also plotted is the best-fit Gaussian, indicating a spin-orbit misalignment of $\lambda=-12^{\circ} \pm 15^{\circ}$. 


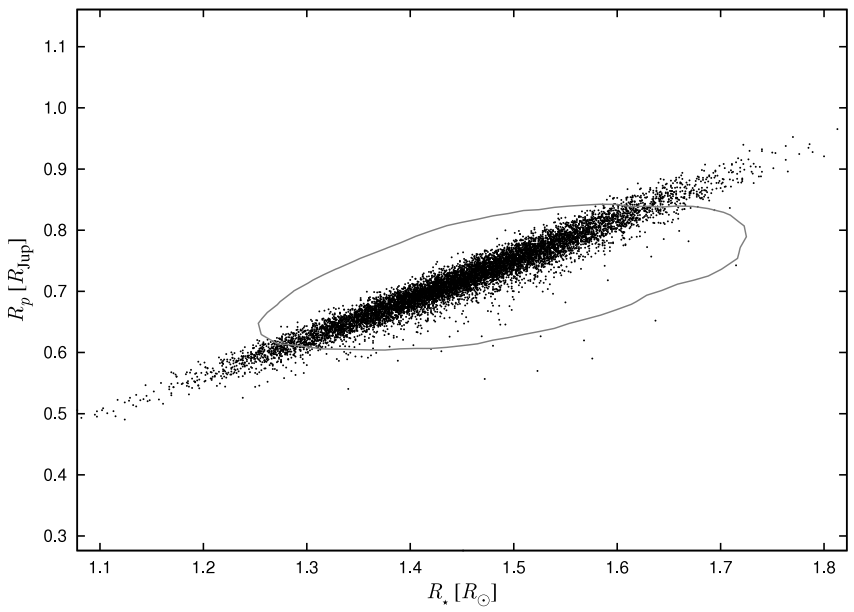

FIG. 5.-Well-known planetary-stellar radius correlation in photometric analyses reflected in the two-dimensional distribution of $10^{4}$ bootstrap Monte Carlo results. The contour shown is the iso- $\chi^{2}$ contour that contains $90 \%$ of the results. This contour only roughly approximates the distribution of Monte Carlo results, as the values of $R_{\star}$ were not fitted, but rather drawn from the Gaussian distribution corresponding to the spectroscopically measured radius of $R_{\star}=1.45 \pm 0.10 R_{\odot}$.

most well-constrained time of central transit, $T_{c}$, and the transit duration, $t_{\mathrm{dur}}$, which refers to the time between the points of first and last contact during ingress and egress, respectively. Of particular interest are the one-dimensional probability distributions for the planetary radius and spin-orbit misalignment. These plots are shown in Figures 3 and 4. We report symmetric error bars for all parameters except $P, M_{0}$, and $v \sin I_{\star}$; the centroids of the distri- butions in these three parameters differ enough from their best-fit values that they warrant asymmetric treatment.

In addition to calculating error bars for each parameter, we also construct and display in Figures 5 and 6 two-dimensional probability distributions for the physically significant model parameters. These plots display two-dimensional projections of the distribution of bootstrap results in 17 dimensional parameter space. Also plotted are the $90 \%$ iso- $\chi^{2}$ contours for each pair of parameters. These contours were obtained by fixing all parameters to their best-fit values for the complete data set and then perturbing the two parameters of interest and calculating $\chi^{2}$. This process generates a set of $\chi^{2}$ contours that are then searched to identify the $\chi^{2}$ value that encloses $90 \%$ of the bootstrap results. From Figure 5, it is clear that errors in the planetary radius, $R_{p}$, are dominated by the uncertainty in the stellar radius, $R_{\star}$, as found previously by Sato et al. (2005). Figure 6 displays the uncertainty distributions for the remaining physically significant parameters. The figure is divided into three regions in order to reflect the primary data type responsible for constraining the fit for each of the model parameters. The upper region displays the uncertainty distributions for the parameters whose values are constrained solely by the RV data: $v \sin I_{\star}, \lambda, M_{p}$, and $P$. Conversely, the right-hand region contains uncertainty distributions for the parameters determined primarily by the photometry: ${ }^{9} R_{p}$, $I$, and $M_{0}$. In both of these regions, the $\chi^{2}$ contours do a decent job of reflecting the distribution of Monte Carlo results, as is expected for parameters that describe a model of a single data type. The central block in

\footnotetext{
9 While it is true that all of these parameters play some role in the combined orbital-Rossiter RV model, their values are determined primarily by the overwhelming amount of photometric data.
}

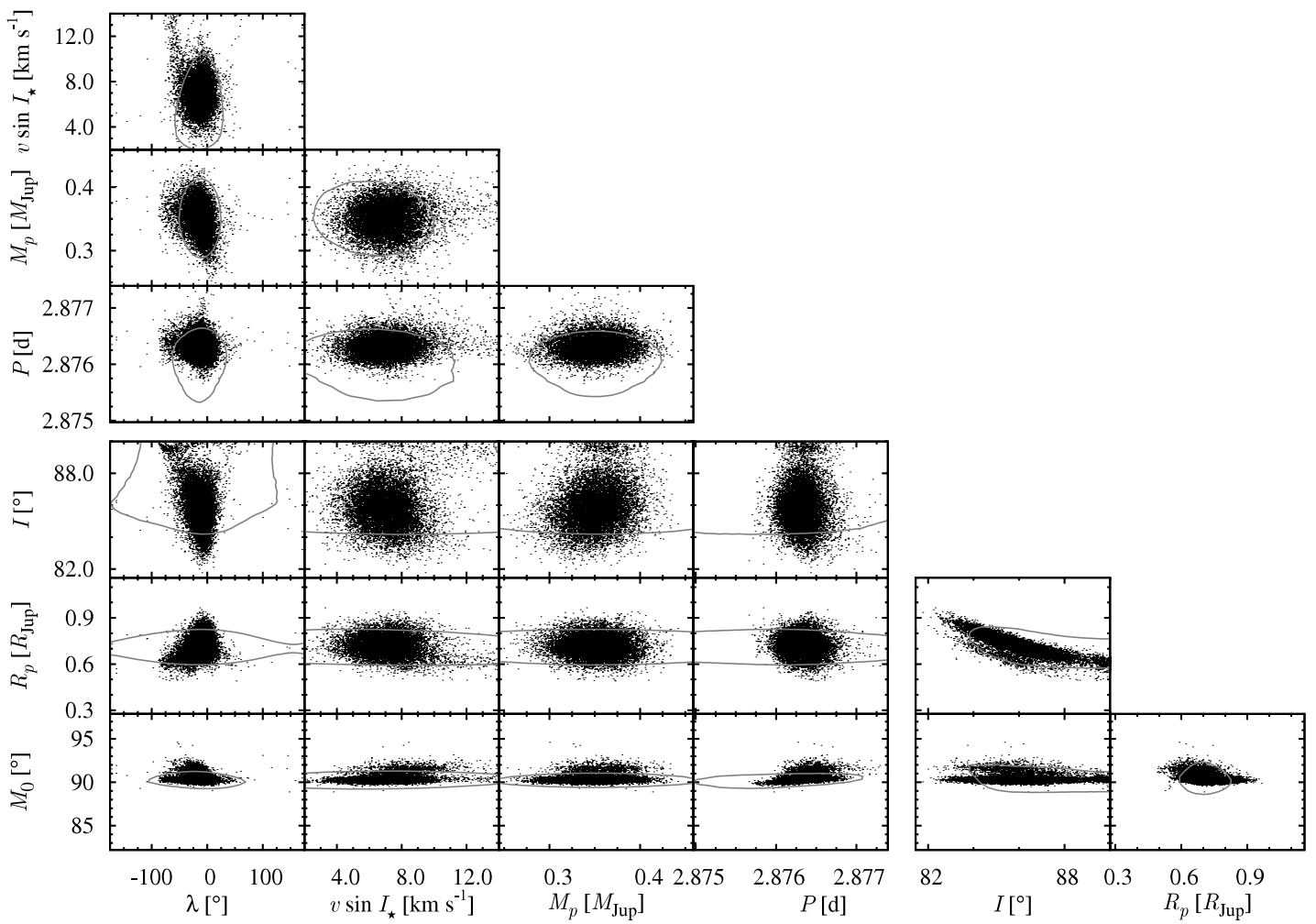

FIG. 6.- Two-dimensional distributions of $10^{4}$ bootstrap Monte Carlo results. All of the physically significant fitted parameters are plotted against one another. The contours shown are the iso- $\chi^{2}$ contours that contain $90 \%$ of the results. This figure is divided into three regions according to the data type that dominates the fit for the plotted parameters. The upper and right-hand regions display the relative uncertainty distributions for RV and photometry-dominated parameters, respectively, while the central block contains the distributions for RV and photometric parameters plotted against one another. 
Figure 6, which plots the RV and photometric parameters against one another, is noticeably different, with few of the contours even remotely approximating curves of equal probability density. In the upper 8 of 12 plots that make up the central block, the $\chi^{2}$ contours extend well beyond the limits of the RV parameter distributions. This behavior is a result of the weak coupling between the photometric and RV components of the model. Since there exist nearly 60 times more photometric data than RV data, equal perturbations away from the best fit for the RV parameters have significantly smaller effect on the global $\chi^{2}$ value than for photometric parameters. The four bottom plots, which show $M_{0}$ against the RV parameters, have somewhat more reasonable contours, since the $\mathrm{RV}$ data does provide some constraint on the mean anomaly, unlike for the other photometric parameters. These complications aside, it is clear from the Monte Carlo distributions that the model is capable of adjusting its two components to obtain the best and most well-constrained self-consistent global fit to the data.

Focusing on the specific subplots of Figure 6, we find that many of the parameters are fairly uncorrelated, with a couple notable exceptions. First, the well-known relationship between $R_{p}$ and $I$ is easily visible; although we do not display the one-dimensional probability distribution for the orbital inclination, $I$, we find a small non-Gaussian cluster of solutions with inclination near $90^{\circ}$, in agreement with the findings of Charbonneau et al. (2006). Similarly, there is a noticeable branch of solutions with earlier central transit times, corresponding (by eq. [2]) to a larger mean anomaly of $M_{0} \sim 92^{\circ}$. This small ambiguity indicates that the times of central transit have not been as tightly constrained as possible. This is likely because all of the transit observations were performed within a time span corresponding to only a few orbits, and a number of them are only partial transits, allowing small adjustments in the period to absorb shifts in the transit centers. This situation will be easily remedied, however, by one or more accurate groundbased observations of full transits many orbits after the current observations. The inclusion of a new photometric data set will shrink the current uncertainties in the period and mean anomaly as well as removing this non-Gaussian group of early transit solutions. In addition, since the perceived asymmetry in the Rossiter curve is sensitive to the determined time of central transit, these new observations, which effectively fix the transit centers and durations, would also likely result in a modest improvement to the uncertainty in the spin-orbit misalignment angle.

\section{DISCUSSION AND CONCLUSION}

When compared with the value $\lambda=-4.4^{\circ} \pm 1.4^{\circ}$ obtained for HD 209458 by Winn et al. (2005), our result for the spin-orbit misalignment, $\lambda=-12^{\circ} \pm 15^{\circ}$, appears to have a rather large uncertainty. While both of these results indicate that the two systems are not significantly misaligned, the value for HD 209458 provides a considerably tighter constraint. The cause of this discrepancy is the relative difference in photometric transit depths for the two planet-star configurations, equivalent to a decrease in the signalto-noise ratio by about a factor of 4 . It is likewise important to note that we report an uncertainty in the central transit time that is slightly more than 2 times larger than that reported in Charbonneau et al. (2006). In order to understand this discrepancy, we performed the Monte Carlo error analysis of the photometry and the seven initial out-of-transit RV measurements as described in Charbonneau et al. Using the Levenburg-Marquardt minimization scheme, we obtained lower $\chi^{2}$ values than those reported for the best fit to the data as well as for the bootstrap Monte Carlo runs. We also obtain larger error bars than those reported in Charbonneau et al., consistent with those reported here. We thus attribute the difference to an inability of the AMEOBA minimization algorithm used by Charbonneau et al. to effectively depart from its initial starting guess when finding the best fit, thereby generating artificially small error estimates. This issue seems to arise when trying to locate the best fit within a noisy and shallow minimum in $\chi^{2}$ space. It is not clear whether this discrepancy has any great significance; however, it will surely be resolved as more data is obtained and parameter estimates improve.

The analysis reported here also verifies the results of Sato et al. (2005) for HD 149026, which reported a planetary mass and radius of $M_{p}=0.36 M_{\text {Jup }}$ and $R_{p}=0.725 R_{\text {Jup }}$. Analysis of the Rossiter curve has also allowed us to determine the stellar rotation speed to be $v \sin I_{\star}=6.2_{-0.6}^{+2.1} \mathrm{~km} \mathrm{~s}^{-1}$, which is consistent with the spectrally determined value of $v \sin I_{\star}=6.0 \pm 0.5 \mathrm{~km} \mathrm{~s}^{-1}$, reported in Sato et al. Most importantly, we have confirmed that the planetary radius $R_{p}=0.718 \pm 0.065 R_{\text {Jup }}$ is indeed remarkably small. The determined mass and radius of the planet imply a mean planetary density of $1.18_{-0.30}^{+0.38} \mathrm{~g} \mathrm{~cm}^{-3}$, which is notably larger than the mean density of Saturn, $0.6873 \mathrm{~g} \mathrm{~cm}^{-3}$. As reported in Sato et al., planetary evolution models show that this small planetary radius implies that the planet has a large fraction of heavy elements in its composition, comprising over $50 \%$ of the planet's total mass. The existence of a core-dominated giant planet such as HD 149026b provides strong evidence that core accretion plays a role in the process of giant planet formation.

With the knowledge that HD 149026b's orbital angular momentum vector is not significantly misaligned with the spin axis of the parent star, we can begin to discern between different plausible formation histories for this unusual planet. With such an anomalous system, one might expect to find clear signatures of a truly strange formation history; however, our measurement of the spin-orbit alignment excludes any exotic scenarios that might excite large misalignments. Unfortunately, the precision provided by the current data cannot discern between the disk interaction and planet embryo collision scenarios, both presented in Sato et al. (2005). In the disk-interaction scenario, planetary migration is halted at the interior 2:1 resonance with the so-called magnetic X-point (see Shu et al. 1994), marking inner disk truncation. In this scenario, the planet underwent differential disk migration until it reached the 2:1 resonance. After being trapped in this resonant configuration, it maintained constant radial position for the remainder of the disk lifetime. As disk material migrated inward through the X-point, the ionized magnetically coupled gas was forced out of the disk, climbing up the magnetic field lines to be fed directly onto the star. The gas-poor material that was left behind in the disk continued to spiral inward, allowing the planet to gather its burden of predominately heavy elements. This more complicated story is compared to a relatively simple one in which two giant planet embryos collided and merged forming a very large heavy element core, which continued to grow by collecting gas for the remainder of the lifetime of the disk. This scenario would affect the alignment angle; however, it would not be capable of producing a final misalignment that is larger than the misalignment of the embryos themselves, and thus cannot produce deviations large enough to be seen with the current precision. Unfortunately, for now the formation of this object must remain a mystery with just one more moderate constraint pointing us toward the answer.

We thank Peter Bodenheimer, David Charbonneau, and Josh Winn for useful discussions. Aaron Wolf's research on this project was supported by the NASA Astrobiology Institute through grant 
NNA 04-CC99A (to G. L.). This research was also supported by NASA grant NNG 04-GK19G and NSF grant AST 04-49986 (to G. L.); NASA grant NNG 05-G164G (to D. A. F.); NASA grant NCC 5-511 and NSF grant HRD 97-06268 (to G. W. H.); NSF grant AST 03-07493 (to S. S. V.); NSF grant AST 99-88087 and travel support from the Carnegie Institution of Washington (to R. P. B.); NASA grant NAG 5-8299 and NSF grant AST 95-20443 (to G. W. M.); and by Sun Microsystems. This research has made use of the Simbad database, operated at CDS, Strasbourg, France. Finally, the authors wish to extend thanks to those of Hawaiian ancestry on whose sacred mountain of Mauna Kea we are privileged to be guests. Without their generous hospitality, the Keck observations presented herein would not have been possible.
Bodenheimer, P., Laughlin, G., \& Lin, D. 2003, ApJ, 592, 555

Boss, A. P. 1997, Science, 276, 1836 2000, ApJ, 536, L101 . 2003, ApJ, 599, 577

Bouchy, F., Pont, F., Santos, N. C., Melo, C., Mayor, M., Queloz, D., \& Udry, S. 2004, A\&A, 421, L13

Bundy, K., \& Marcy, G. W. 2000, PASP, 112, 1421

Butler, R. P., Marcy, G. W., Williams, E., McCarthy, C., Dosanjh, P., \& Vogt, S. S. 1996 , PASP, 108,500

Charbonneau, D. 2004, in IAU Symp. 219, Stars as Suns: Activity, Evolution, and Planets, ed. A. K. Dupree \& A. D. Benz (San Francisco: ASP), 366

Charbonneau, D., et al. 2006, ApJ, 636, 445

Fischer, D., et al. 2005, ApJ, 620, 481

Fortney, J. J., Sanmon, D., Marley, M. S., Lodders, K., \& Freedman,R. 2006, ApJ, 642, 495

Holman, M. J., Winn, J. N., Stanek, K. Z., Torres, G., Sasselov, D. D., Allen, R. L., \& Fraser, W. 2007, ApJ, 655, 1103

Hubickyj, O., Bodenheimer, P., \& Lissauer, J. J. 2004, Rev. Mex. A\&A, 22, 83

Laughlin, G., Marcy, G. W., Vogt, S. S., Fischer, D. A., \& Butler, R. P. 2005, ApJ, 629, L121

McLaughlin, D. D. 1924, ApJ, 60, 22

\section{REFERENCES}

Ohta, Y., Taruya, A., \& Suto, Y. 2005, ApJ, 622, 1118

Moutou, C., Pont, F., \& Mayor, M. 2004, A\&A, 424, L31

Pollack, J. B., et al. 1996, Icarus, 124, 62

Pont, F., Bouchy, F., Queloz, D., Santos, N. C., Melo, C., Mayor, M., \& Udry, S. 2004, A\&A, 426, L15

Press, W. H., Teulkiolsky, S. A., Vetterling, W. T., \& Flannery, B. P. 1992, Numerical Recipes in Fortran: The Art of Scientific Computing (2nd ed; Cambridge: Cambridge Univ. Press)

Queloz, D., Eggenberger, A., Mayor, M., Perrier, C., Beuzit, J. L., Naef, D., Sivan, J. P., \& Udry, S. 2000, A\&A, 359, L13

Rossiter, R. A. 1924, ApJ, 60, 15

Saar, S. H., Butler, R. P., \& Marcy, G. W. 1998, ApJ, 498, L153

Sato, B., et al. 2005, ApJ, 633, 465

Shu, F., Najita, J., Ostriker, E., Wilkin, F., Ruden, S., \& Lizano, S. 1994, ApJ, 429, 797

Vogt, S. S., et al. 1994, Proc. SPIE 2198, 362

Winn, J., et al. 2005, ApJ, 631, 1215

Wittenmyer, R. A., et al. 2005, ApJ, 632, 1157

Wright, J. T., Marcy, G. W., Butler, R. P., \& Vogt, S. S. 2004, ApJS, 152, 261 